\title{
Hyperion Plasma FIB for Precision 3D TSV Analysis
}

\author{
Noel S Smith ${ }^{1}$, Paul P Tesch ${ }^{1}$, Noel P Martin ${ }^{1}$ and Rod W Boswell ${ }^{1,2}$ \\ ${ }^{1}$ Oregon Physics LLC, 2704 SE 39th Loop, Suite 109, Hillsboro, OR 97123 \\ 2 Australian National University, Oliphant Building 60, Mills Road, Canberra, ACT 0200, Australia
}

While advances in conventional CMOS scaling inevitably slows down and departs from the exponential trend known as Moore's law, there is further focus on advancing system integration to provide smaller devices, with greater functionality and less power consumption. The combination of die stacking with direct Through-Silicon-Via (TSV) interconnects, appears to be emerging as the most promising solution [1]. Although 3D stacked die and TSV interconnects promise significant performance advances, the development of economical and reliable TSV manufacturing methods is proving to be a significant challenge [2].

A key element to shortening the learning curve for TSV development, is the ability to carry out rapid, high precision inspection and metrology of the vias. Some of the features that require inspection include critical via dimensions, sidewall profiles, barrier and seed layer thickness and coverage, metallic grain size distributions, layer delamination and any voids left during the final filling process. TSV analysis often requires an ability to detect and measure features and defects with dimensions ranging from $10 \mathrm{~nm}$ to $20 \mathrm{um}$, within $20-600 \mathrm{um}$ long vias. Here we demonstrate how the Hyperion ${ }^{\mathrm{TM}}$ Plasma Focused Ion Beam (FIB) $[3,4]$, coupled with a conventional gallium FIB for in-situ imaging, provides rapid analysis speed and high resolution 3D inspection of TSV's (fig 1.). Since the Hyperion ${ }^{\mathrm{TM}}$ FIB extends FIB milling speeds up to 1000 's $\mu^{3} / \mathrm{s}$, while maintaining the ability to sequentially slice and image with nanometer scale precision, this method of analysis is proving to be significant in the development of 3D IC interconnects.

Gallium FIB tools stand alone in their ability to efficiently mill material surfaces with sub-100nm precision and with milling speeds in the $0-5 \mu \mathrm{m}^{3} / \mathrm{s}$ [5]. However, this is often too slow for crosssectioning packaging and interconnect structures that can have linear dimensions of $>30 \mu \mathrm{m}$. These relatively large TSV structures require focused beams with sufficient beam current to remove 1000's $\mu \mathrm{m} / \mathrm{s}$, to enable rapid interconnect cross-sectioning and subsequent analysis (fig 2.). Today, the Hyperion $^{\mathrm{TM}}$ Plasma-FIB has extended the milling speed of the FIB technique by a factor of 100 and is routinely used for TSV slicing and imaging.

Here we demonstrate the capability of the Hyperion ${ }^{\mathrm{TM}}$ Plasma-FIB system for rapid, multiperspective and ultra high resolution TSV analysis.

\section{References}

[1] M. Motoyoshi, Proceedings of the IEEE, Vol. 97, No.1, 43 (Jan 2009)

[2] S. Golovato, G. Seryogin, and D. Goodman, Chip Scale Review, 18, (Jan 2010)

[3] N. Smith, P. Tesch, N. Martin and R. Boswell, Microscopy Today,32 (Sept. 2009)

[4] P Tesch, N Smith, N Martin, D Kinion, ISTFA Conf Proc, 7 (2008)

[5] L.A. Giannuzzi and F.A. Stevie, Introduction to Focused Ion Beams, Springer (2005). 


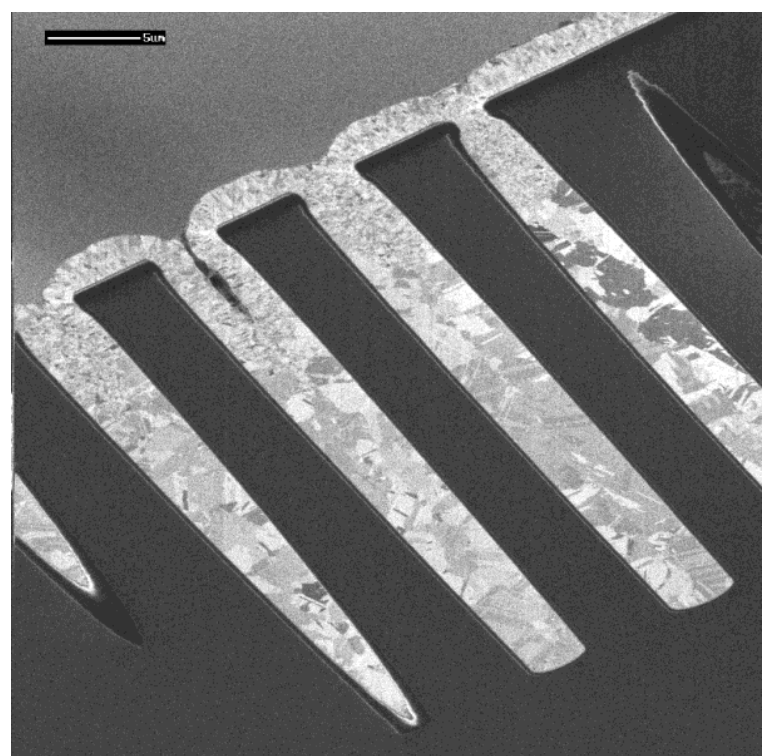

FIG. 1. TSV's sectioned with Hyperion FIB and imaged with conventional gallium FIB.

Vias produced at IMEC during fabrication process development.

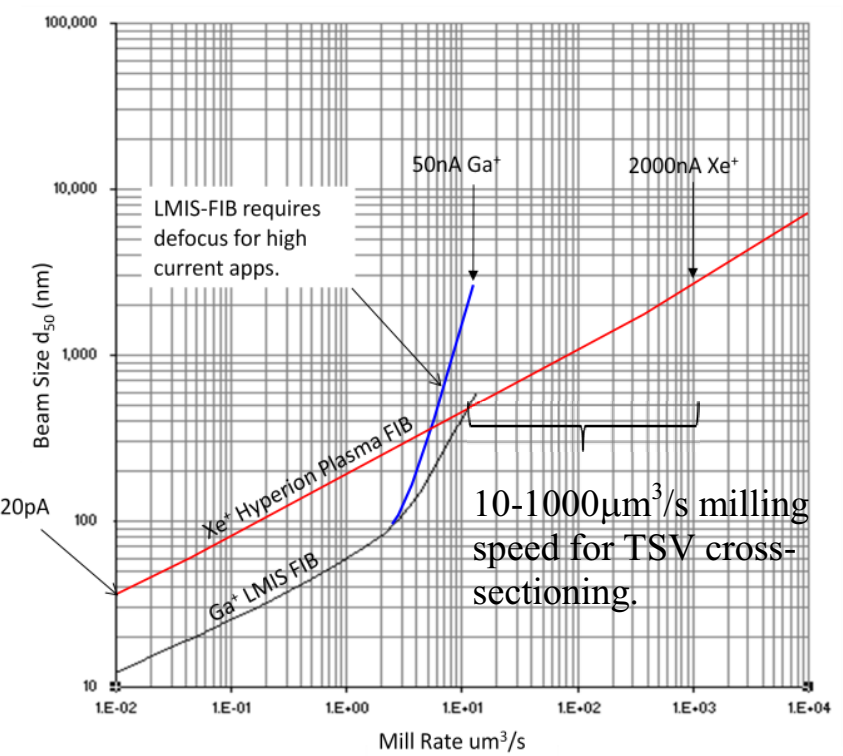

FIG. 2. Beam size versus milling rate (milling silicon at normal incidence) 